\title{
COMPONENTES CLÍNICO-EPIDEMIOLÓGICOS DE CRIANÇAS E ADOLESCENTES EM HEMODIÁLISE*
}

Reginaldo Passoni dos Santos¹, Daniele Laís Brandalize Rocha², Vera Hermina Kalika Koch ${ }^{3}$

1Enfermeiro. Pontifícia Universidade Católica. Toledo-PR-Brasil.

Enfermeira. Mestre em Enfermagem. Pontifícia Universidade Católica. Toledo-PR-Brasil.

${ }^{3}$ Médica. Doutora em Medicina. Universidade de São Paulo. São Paulo-SP-Brasil.

RESUMO: Este estudo objetivou identificar os componentes clínico-epidemiológicos de crianças e adolescentes em hemodiálise e foi realizado por meio de investigação documental. Foram analisados os prontuários de todos os pacientes que realizavam hemodiálise em um hospital pediátrico do Estado do Paraná-Brasil em janeiro de 2013, utilizando um roteiro semiestruturado para o registro das informações relativas às variáveis clínico-epidemiológicas. Os resultados da análise de 22 prontuários apontaram distribuição homogênea entre os gêneros, sendo que 59,1\% tinham entre 11 e 15 anos, 86,1\% tempo de permanência em hemodiálise de até dois anos e 59,1\% fístula arteriovenosa como via de acesso vascular. As causas mais prevalentes para a doença renal crônica foram anomalias congênitas do trato urológico (45,5\%) e hipertensão arterial sistêmica (31,8\%). Os resultados possibilitam especificar e direcionar ações de prevenção e de cuidados de enfermagem e de saúde. DESCRITORES: Diálise renal ; Saúde da criança; Saúde do adolescente; Epidemiologia; Enfermagem.

\section{CLINICAL-EPIDEMIOLOGICAL COMPONENTS OF CHILDREN AND ADOLESCENTS IN HEMODIALYSIS}

\begin{abstract}
This study aimed to identify the clinicalepidemiological aspects of children and adolescents receiving hemodialysis and was undertaken through a documental investigation. The hospital records of all the patients who received hemodialysis in a pediatric hospital in the state of Paraná, Brazil, in January 2013 were analyzed, using a semistructured script for recording information relating to the clinical-epidemiological variables. The results of the analysis of 22 hospital records indicated a homogenous distribution between the sexes, with $59.1 \%$ aged between 11 and 15 years old, 86.1\% having received hemodialysis for up to two years, and $59.1 \%$ with the arteriovenous fistula (AVF) as the vascular access. The most prevalent causes for chronic kidney disease were congenital anomalies of the urinary tract $(45.5 \%)$ and systemic hypertension $(31.8 \%)$. The results allow the specification and directing of preventive, nursing and health actions.
\end{abstract}

DESCRIPTORS: Renal dialysis; Child's health; Adolescent's health; Epidemiology; Nursing.

\section{COMPONENTES CLÍNICO-EPIDEMIOLÓGICOS DE NIÑOS Y ADOLESCENTES EN HEMODIÁLISIS}

RESUMEN: Este estudio tuvo el objetivo de identificar los componentes clínico-epidemiológicos de niños y adolescentes en hemodiálisis y fue realizado por medio de investigación documental. Fueron analizados los prontuarios de todos los pacientes que realizaban hemodiálisis en un hospital pediátrico del estado de Paraná, Brasil, en enero de 2013, utilizándose un guión semiestructurado para el registro de las informaciones referentes a las variables clínico-epidemiológicas. Los resultados del análisis de 22 prontuarios apuntaron distribución homogénea entre los géneros, siendo que 59,1\% tenían entre 11 y 15 años, 86,1\% tiempo de permanencia en hemodiálisis hasta dos años y $59,1 \%$ fístula arteriovenosa como vía de acceso vascular. Las causas más prevalentes para la enfermedad renal crónica fueron anomalías congénitas del aparato trato urológico (45,5\%) e hipertensión arterial sistémica (31,8\%). Los resultados posibilitan especificar y direccionar acciones de prevención y de cuidados de enfermería y de salud.

DESCRIPTORES: Diálisis renal; Salud del niño; Salud del adolescente; Epidemiología; Enfermería.

*Artigo extraído do Trabalho de Conclusão Do Curso de Graduação em Enfermagem apresentado

à Pontifícia Universidade Católica do Paraná, campus Toledo, em 2013.

Recebido: $17 / 10 / 2013$ Finalizado: 15/03/2014

Autor correspondente:

Reginaldo Passoni dos Santos

Pontifícia Universidade Católica do Paraná

Avenida da União, 500 - 85902532 Toledo-PR-Brasil

E-mail: regi-pas@hotmail.com 


\section{INTRODUÇÃO}

A Doença Renal Crônica (DRC) configura-se como uma afecção irreversível e silenciosa que atinge milhões de pessoas em todo o mundo. Para que uma pessoa seja diagnosticada como portadora de DRC, é preciso haver a apresentação de dano renal ou Taxa de Filtração Glomerular (TFG) $<60 \mathrm{~mL} / \mathrm{min} . / 1,73 \mathrm{~m} 2$ por, no mínimo, três meses consecutivos ${ }^{(1)}$. Com frequência, a percepção da função renal diminuída é facilmente identificada determinando-se o nível de marcadores da doença presentes no sangue e na urina, bem como por meio de estudo de imagem. Contudo, destaca-se que para crianças e adolescentes a TFG deve ser determinada a partir de uma equação que leva em conta a concentração sérica de creatinina, a altura e o gênero no paciente, uma vez que o nível de creatinina sérica por si só não deve ser utilizado para avaliação da função renal em pediatria ${ }^{(1)}$.

Devido ao fato de a diminuição da TFG ser progressiva e lenta, não raro a DRC só é percebida quando já houve no indivíduo uma perda renal funcional de mais de $50 \%$. Dessa forma, uma TFG $<30 \mathrm{~mL} / \mathrm{min} . / 1,73 \mathrm{~m} 2$ indica função renal severamente comprometida e o paciente deve ser avaliado para dar entrada em um programa de Terapia Renal Substitutiva (TRS) ${ }^{(1)}$.

Apresentam-se atualmente três modalidades de TRS: diálise peritoneal (DP), hemodiálise (HD) e transplante renal (Tx renal). Apesar de a HD ser a terapêutica predominante entre pacientes com DRC em TRS, a escolha da modalidade mais adequada irá depender de diversos fatores, sendo que a identificação do estágio da doença, estabelecida conforme diretrizes elaboradas pela National Kidney Foundation, apresenta-se como subsídio à tomada de decisão acerca da modalidade mais indicada para cada paciente ${ }^{(1-2)}$.

Destaca-se, que a TRS é significativamente onerosa aos cofres públicos, em especial o tratamento hemodialítico. No Brasil, mais de $80 \%$ dos pacientes em TRS obtêm seu financiamento por meio do Sistema Único de Saúde, o que acarreta um gasto de milhões de reais todos os $\operatorname{anos}^{(3)}$.

Apesar de não exigir internação, em muitos lugaresa HD ocorre dentro de uma unidade hospitalar e isso acaba por ocasionar "[...] grande tensão psicológica para a criança/adolescente [...]"(4:19). Por este motivo, a assistência ao paciente deve ser realizada por uma equipe multiprofissional visando a atender todas as necessidades apresentadas ${ }^{(5)}$.

Nesse contexto de multiprofissionalismo para o cuidado, o papel do enfermeiro é, dentre outros, estabelecer estratégias para a Sistematização da Assistência de Enfermagem (SAE) à criança e ao adolescente em HD, de forma a abarcar ações a serem desenvolvidas por sua equipe que visam não apenas a qualidade técnico-científica da assistência, mas também a promoção da qualidade de vida dos pacientes assistidos através de intervenções específicas e direcionadas ${ }^{(6-7)}$. Para tanto, é necessário, primeiramente, que a equipe conheça as características próprias de seu público, tendo em vista que pacientes pediátricos em HD possuem características peculiares, frequentemente, adversas daquelas percebidas na população adulta( ${ }^{(8)}$.

Concernente ao disposto, em Nefrologia, assim como nas demais ciências da saúde, a recuperação do paciente deixou a muito de depender apenas de ações curativas. Aponta-se como de grande importância que todo profissional de saúde - independente do campo de atuação - busque em evidências científicas o pilar subsidiário ao exercício profissional desenvolvido a partir de práticas assistenciais inovadoras, que viabilize ofertar ao paciente a completa recuperação de seu bem-estar físico, mental e social da forma mais antecipada possível ${ }^{(9)}$.

Entretanto, um número bastante limitado de estudos acerca do perfil clínico e epidemiológico do público infanto-juvenil portador de DRC em tratamento hemodialítico é citado, havendo real necessidade de tal conhecimento para a oferta do cuidado de maneira holística e efetiva ${ }^{(10)}$.

Frente ao apresentado, bem como à relevância social, acadêmico-científica e profissional que permeia sobre o assunto, caracterizouse como objetivo deste estudo identificar os componentes clínico-epidemiológicos de crianças e adolescentes portadores de DRC em tratamento hemodialítico, assistidas em um hospital pediátrico no Estado do Paraná-Brasil.

\section{MÉTODO}

Trata-se de uma pesquisa segue a abordagem quantitativa, com investigação descritiva e do tipo documental. Com relação ao ambiente do estudo, elegeu-se a unidade de hemodiálise de 
um serviço de nefropediatria de um hospital de grande porte, na cidade de Curitiba, Paraná, o qual é referência nacional no atendimento de alta complexidade à crianças e adolescentes. A amostragem foi composta pelos prontuários de todos os pacientes em tratamento hemodialítico quando da coleta de dados, realizada durante o mês de janeiro de 2013 por meio da utilização de um roteiro semiestruturado.

Foram coletados dados referentes aos seguintes eixos de análises: identificação do paciente (variáveis: gênero, faixa etária e procedência geográfica); diagnóstico clínico (variável: causa básica da DRC); seguimento em hemodiálise (variáveis: tempo de tratamento hemodialítico e idade no início da TRS) e dados clínicos (variáveis: creatinina sérica no início da TRS, comorbidades e via de acesso vascular). Os dados de todas as variáveis foram analisados por meio de suas freqüências absolutas e relativas com a utilização

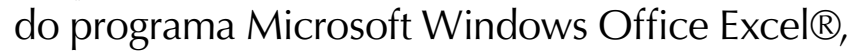
versão 2010, apresentando-se os resultados de modo descritivo e na forma de tabelas.

Em respeito aos princípios éticos das pesquisas envolvendo seres humanos estabelcidos pela Resolução 466/2012, este estudo teve seu projeto de pesquisa aprovado pelo Comitê de Ética em Pesquisa da instituição na qual foi realizado, com emissão de parecer favorável sob n. 179.725 em dezembro de 2012.

\section{RESULTADOS}

Realizou-se análise e extração de dados de 22 prontuários, dos quais se pode observar que 50\% $(n=11)$ eram de pacientes do gênero masculino e $50 \%(n=11)$ do gênero feminino; com idade média \pm desvio padrão de 11,5 $\pm 3,8$ anos. A faixa etária e procedência dos pacientes são apresentadas na Tabela 1.

Os dados relativos à causa básica (primária) da DRC apontaram que 45\% ( $n=10)$ dos pacientes desenvolveram a doença devido a alguma anomalia urológica ou má formação congênita; as causas estão apresentadas na Tabela 2.

Já em relação às comorbidades, sete(31,8\%) prontuários apontavam que o paciente apresentava hipertensão arterial sistêmica, três(13,6\%) mielomeningocele e um(4,5\%) fibrose hepática. No restante dos prontuários, ou seja, em 11(50\%) não havia apontamento de afecção secundária.

Com relação à idade dos pacientes no início do tratamento hemodialítico, estes apresentaram idade média \pm desvio padrão de $12,3 \pm 10,3$ meses; os valores da média, mediana e máximomínimo dos níveis séricos de creatinina $(\mathrm{mg} / \mathrm{dL})$ foram: \pm 7,9, 7,0 e 20,1 - 2,8 para meninos; $6,2,5,8$ e 11,1 - 3,2, para meninas. Dados do seguimento na modalidade de tratamento e acesso vascular estão apresentados na Tabela 3.

Tabela 1- Distribuição dos pacientes em hemodiálise segundo gênero, faixa etária e procedência geográfica. Curitiba-PR-Brasil, 2013

\begin{tabular}{lcccc} 
Variável & Homens & Mulheres & $\mathbf{n}$ & $\mathbf{\%}$ \\
\hline Gênero & 11 & 11 & 22 & 100 \\
\hline Faixa etária & & & 2 & 9,1 \\
\hline$\leq 05$ anos & 2 & 0 & 5 & 22,7 \\
\hline 6 a 10 anos & 2 & 3 & 13 & 59,1 \\
\hline 11 a 15 anos & 7 & 6 & 2 & 9,1 \\
\hline$\geq 16$ anos & 0 & 2 & 18 & 81,8 \\
\hline Procedência geográfica & & & 4 & 18,2 \\
\hline Curitiba & 9 & 9 & 0 & 0 \\
\hline Outras cidades do Paraná & 2 & 2 & & \\
\hline Outro Estado & 0 & 0 & & \\
\hline
\end{tabular}


Tabela 2 - Distribuição dos pacientes em hemodiálise segundo causa básica da Doença Renal Crônica. Curitiba- PR-Brasil, 2013

\begin{tabular}{lcccc} 
Anomalia urológica / & & & & Total \\
má formação congênita & Homens & Mulheres & $\mathbf{n}$ & \% \\
\hline Refluxo vésico-ureteral & 3 & 1 & 4 & 18,2 \\
\hline Bexiga neurogênica & 0 & 3 & 3 & 13,6 \\
\hline Válvula de uretra posterior & 2 & 0 & 2 & 9,1 \\
\hline Estenose de junção uretero-piélica & 0 & 1 & 10 & 4,5 \\
\hline Total & 5 & 5 & 3 & 45,5 \\
\hline Outras causas & & & 1 & 13,6 \\
\hline Glomerulonefrite crônica & 1 & 2 & 4 & 4,5 \\
\hline Síndrome nefrótica & 1 & 0 & 1 & 18,2 \\
\hline Doença Renal Crônica não & 2 & 2 & 1 & 4,5 \\
específica & & & 1 & 4,5 \\
\hline Doença Renal Crônica por aids & 1 & 0 & 1 & 4,5 \\
\hline Poliartrite trombolítica & 0 & 1 & 12 & 4,5 \\
\hline Tubulopatia & 1 & 0 & 1 & 54,5 \\
\hline Rins policísticos & 0 & 6 & & \\
\hline Total & 6 & & 1 & \\
\hline
\end{tabular}

Tabela 3 - Distribuição dos pacientes em hemodiálise segundo características clínicas. Curitiba-PR-Brasil, 2013

\begin{tabular}{|c|c|c|c|c|}
\hline Variáveis & Homens & Mulheres & $\mathbf{n}$ & $\%$ \\
\hline \multicolumn{5}{|c|}{ Idade no início da hemodiálise } \\
\hline$\leq 05$ anos & 2 & 0 & 2 & 9,1 \\
\hline 6 a 10 anos & 4 & 5 & 9 & 40,9 \\
\hline 11 a 15 anos & 5 & 5 & 10 & 45,5 \\
\hline$\geq 16$ anos & 0 & 1 & 1 & 4,5 \\
\hline \multicolumn{5}{|c|}{ Tempo de permanência em hemodiálise } \\
\hline$\leq 06$ meses & 2 & 5 & 7 & 31,8 \\
\hline 7 a 12 meses & 4 & 4 & 8 & 36,4 \\
\hline 13 a 18 meses & 2 & 0 & 2 & 9,1 \\
\hline 19 a 24 meses & 1 & 1 & 2 & 9,1 \\
\hline$>24$ meses & 2 & 1 & 3 & 13,6 \\
\hline \multicolumn{5}{|l|}{ Acesso vascular } \\
\hline FAV & 5 & 8 & 13 & 59,1 \\
\hline $\mathrm{CVC}$ & 6 & 3 & 9 & 40,9 \\
\hline \multicolumn{5}{|c|}{ Creatinina (mg/dl) no início da hemodiálise } \\
\hline Média & 7,9 & 6,2 & - & - \\
\hline Mediana & 7,0 & 5,8 & - & - \\
\hline Máximo-Mínimo & $20,1-2,8$ & $11,1-3,2$ & - & - \\
\hline
\end{tabular}




\section{DISCUSSÃO}

Apesar de outras pesquisas realizadas no Brasil $^{(7,11)}$ apontarem predomínio do gênero masculino entre o público pediátrico com DRC, os resultados obtidos neste estudo não evidenciaram essa mesma supremacia, sendo que a distribuição de pacientes, segundo o gênero, foi de $50 \%$. Por outro lado, a faixa etária predominante na presente pesquisa mostrou-se consoante ao apontado na literatura pertinente, bem como pela Associação Brasileira dos Centros de Diálise e Transplante, a qual descreve que pacientes com idade entre 10 e 15 anos apresentaram-se em maior número nos centros pediátricos de TRS do Brasil $^{(12)}$.

Com relação à procedência geográfica, mesmo que os resultados tenham apontado que a maioria dos pacientes era da própria cidade na qual realizavam a TRS, uma vez que em apenas quatro $(18,2 \%)$ prontuários constava outra localidade, vale ressaltar que devido ao fato de o tratamento hemodialítico exigir que o paciente esteja no centro de tratamento, no mínimo, três dias por semana, aliado às horas em que fica nas sessões, faz com que esse se torne extremamente cansativo e, muitas vezes, inviável o deslocamento geográfico longo. Como resultado, pacientes oriundos de outras localidades, encaminhados a serviços de referência, são forçados a fixarem moradia na própria cidade do tratamento.

Do mesmo modo que os resultados relacionados à faixa etária, os dados oriundos da busca pela causa básica da DRC no público investigado ratificaram o apresentado em pesquisas congêneres, evidenciando que as anomalias congênitas se mostram como causa primária de maior supremacia entre a população pediátrica, tendo a HAS como doença secundária prevalente entre os diagnósticos identificados neste levantamento.

Em um departamento de nefrologia pediátrica belga as anomalias congênitas do rim e trato urológico também emergiram como principais causas de DRC em seus pacientes, representando $59 \%$ do total dos $\operatorname{casos}^{(13)}$. Em pesquisa realizada com 331 registros de pacientes da Sérvia e Montenegro, observou-se este mesmo predomínio como principal fator desencadeante para DRC em crianças e adolescentes daquele país. Os autores do referido estudo descreveram ainda, que a idade média dos pacientes no momento do início do tratamento clínico foi de nove anos, ao passo que neste estudo a idade ficou predominantemente na faixa dos seis aos 15 anos. Já o tempo médio de seguimento clínico encontrado foi de quatro anos, tempo bem maior ao emergido neste levantamento, no qual se identificou um número maior de pacientes em HD por um período inferior a um ano ${ }^{(14)}$.

Com referência ao nível sérico de creatinina $(\mathrm{Cr})$, identificou-se aqui apenas a dosagem quando da entrada no programa hemodialítico. Em um estudo $^{(7)}$, a Cr média no momento do diagnóstico clínico de DRC foi igual a 3,0 mg/dL e a TFG por volta de 27,4 (mL/min/1,73m2). Em outro estudo $^{(14)}$ semelhante, a TFG média dos sujeitos foi de 39,6 (mL/min/1,73m2). A presença constante de taxas elevadas de resíduos bio-metabólicos acarreta no paciente renal crônico diversos riscos, principalmente, cardiovasculares. Desse modo, apesar de o tratamento hemodialítico constituirse como a modalidade de última escolha ao paciente pediátrico portador de DRC, quando há azotemia, ou seja, aumento excessivo de excretas biometabólicos, tal como a creatinina, dar ínicio a HD apresenta-se necessário, pois, possibilita o alcance do equilíbrio bioquímico de modo rápido e eficaz, daí sua indicação tambem em situações $\operatorname{agudas}^{(7)}$.

O Projeto Medidas de Desempenho Clínico realizado nos Estados Unidos possibilitou a constatação do predomínio de CVC como via prevalente entre o público avaliado, sendo observado em $61 \%$ dos pacientes ${ }^{(15)}$. Em relatório divulgado por um banco de dados norteamericano é possível observar que, em 2011, $78,7 \%$ dos pacientes pediátricos em HD utilizavam o CVC como via de acesso(16). O predomínio dessa mesma via também foi identificado em pesquisa desenvolvida nas Minas Gerais-Brasil ${ }^{(17)}$. Os autores daquele estudo mencionam que, tal fato relaciona-se em muitos casos à falta de expertise profissional para a confecção de FAV, principalmente, em crianças com comprometimento vascular, uma vez que não é todo centro de TRS que possui atedimento apenas ao público pediátrico.

Neste estudo, observou-se que a via de acesso predominante foi a FAV, conforme apresentado na Tabela 3. Tal dado revela a qualificação e 
habilidade técnica na condução e manejo clínicoassistencial desses pacientes, realizada pela equipe multiprofissional do serviço em questão, pois, evidências científicas apontam diversas dificuldades apresentadas por centros de TRS na busca pela manutenção da perviedade da FAV em pacientes pediátricos ${ }^{(17)}$.

Além disso, a utilização do CVC pode aumentar em até 20 vezes a possibilidade da criança e adolescente em HD contrair infecção, sendo esta a segunda principal causa de mortalidade entre o referido público ${ }^{(10,18)}$. Sabendo disso, figura-se como atribuição da equipe de enfermagem o estabelecimento de condutas que visem à prevenção de infecções naqueles pacientes cuja via de acesso se dá pelo CVC, dentre elas, a adoção de medidas de assepsia e anti-sepssia no momento de conexão/ desconexão no dialisador ${ }^{(18)}$.

Aos que utilizam a FAV, os cuidados essenciais se relacionam com prevenção de hemorrágias, traumas locais, obstrução venosa, e outros ${ }^{(19)}$. $\mathrm{O}$ enfermeiro e equipe de enfermagem também devem estar atentos às intercorrências durante o procedimento hemodialítico, oriundas, especialmente, de desequilíbrio hidroeletrolítico e ácido-básico, instabilidade hemodinâmica e dor aguda. Tãnto quanto ofertar um cuidado humanizado, ter amplo conhecimento técnicocientífico especializado mostra-se de fundamental importância para o desenvolvimento da SAE de forma a evitar imperícias e imprudências ${ }^{(6,18)}$.

\section{CONCLUSÃO}

Os resultados deste estudo assemelhamse, com resultados de pesquisas congêneres realizadas no Brasil e no mundo. A realização do presente estudo possibilitou aos pesquisadores elencarem determinadas conclusões inerentes ao perfil clínico-epidemiológico de crianças e adolescentes em hemodiálise, a saber: má formação congênita do trato urológico como principal diagnóstico primário; prevalência de HAS secundária à doença de base; faixa etária com predomínio de público pré/adolescente; instalações de residência preponderantemente na cidade de Curitiba.

Conhecer o perfil do público infanto- juvenil em hemodiálise possibilita adquirir subisídios necessários à elaboração de estratégias assistenciais específicas e direcionadas às necessidades peculiares dessa clientela, além de contribuir para o desenvolvimento de intervenções expansíveis ao se agregar resultados de diversos estudos congêneres.

Considera-se também que, devido à equipe de enfermagem desempenhar importante papel assistencial e educativo junto a este público e seus familiares, a abordagem dos pacientes com questões pouco difundidas, como a sexualidade, poderá melhorar a relação profissional-paciente.

\section{REFERÊNCIAS}

1. National Kidney Foundation. K/DOIQ clinical practice guidelines for chronic kidney disease in children and adolescents: evaluation, classification, and stratification. Pediatrics. 2003;111(6):1416-21.

2. Sociedade Brasileira de Nefrologia [Internet]. Doenças comuns: Insuficiência renal. São Paulo: SBN. [acesso em 01 out 2012]. Disponível: http://www.sbn.org.br/ leigos/index.php?insuficienciaRenal\&menu=24.

3. Oliveira MB, Junior JER, Zatz R. End-Stage renal disease in Brazil: Epimiology, prevention and treatment. Kidney International. 2005;68(Sppl. 97):82-6.

4. Abrahão SS, Ricas J, Andrade DF, Pompeu FC, Chamahum L, Araújo TM et al. Difficulties experienced by children/adolescents with chronic kidney disease and by their families. J. Bras. Nefrol. [Internet]. 2010;32(1) [acesso em 02 out 2012. Disponível: http:// dx.doi.org/10.1590/S0101-28002010000100004

5. Bastos MG, Kirstajn GM. Chronic kidney disease: importance of early diagnosis, immediate referral and structured interdisciplinary approach to improve outcomes in patients not yet on dialysis. J. Bras. Nefrol. [Internet]. 2011;33(1):93-108. [acesso em 01 out 2012. Disponível: http://dx.doi.org/10.1590/S010128002011000100013

6. Moreira DS, Vieira MRR. Crianças em tratamento dialítico: a assistência pelo enfermeiro. Arq. Ciênc Saúde. [Internet]. 2010;17(1):27-34. [acesso em 02 out 2012]. Disponível: http://www.cienciasdasaude. famerp.br/racs_ol/vol-17-1/IDL4_jan-mar_2010.pdf.

7. Zorzo RA. Perfil clínico-epidemiológico de 121 crianças e adolescentes com doença renal crônica: 22 anos de experiência do Hospital das Clínicas da Faculdade de Medicina de Ribeirão Preto da Universidade de São Paulo. [Dissertação]. Ribeirão Preto: Faculdade de Medicina/USP; 2008. 99p. 
[Internet] [acesso em 02 out 2012]. Disponível: http:// www.teses.usp.br/teses/disponiveis/17/17138/tde28072008-211159/pt-br.php.

8. Pennafort VPS, Queiroz MVO. Componentes clínicos associados aos cuidados de enfermagem a crianças e adolescentes com doença renal crônica. Rev Rene. [Internet]. 2011;12(4) [acesso em 02 out 2012]. Disponível: http://www.revistarene.ufc.br/revista/ index.php/revista/article/view/294/pdf.

9. Pedrolo E , Danski MTR, Mingorance P, Lazzari LSM, Méier MJ, Crozeta K. A prática baseada em evidências como ferramenta para a prática profissional do enfermeiro. Cogitare enferm. [Internet]. 2009;14(4) [acesso em 16 out 2012]. Disponível: http:// ojs.c3sl.ufpr.br/ojs2/index.php/cogitare/article/ view/16396/10875.

10. Harambat J, Stralen KJV, Kim JJ, Tizard EJ. Epidemiology of chronic kidney disease in children. Pediatr. Nephrol. [Internet]. 2011;27(3) [acesso em 15 out 2012]. Disponível: http://www.ncbi.nlm.nih.gov/pmc/articles/ PMC3264851/. doi:10.1007/s00467-011-1939-1

11. Nogueira PCK, Feltran LS, Camargo MF, Leão ER, Benninghoven JRCS, Gonçalves NZ, et al. Prevalência estimada da doença renal crônica terminal em crianças no estado de São Paulo. Rev. Assoc. Med. Bras. [Internet] 2011;57(4) [acesso em 15 out 2012]. Disponível: http://dx.doi.org/10.1590/S010442302011000400020

12. Associação Brasileira dos Centros de Diálise e Transplante [Internet]. Criança também sobre de insuficiência renal. Brasília: ABCDT. [acesso em 15 out 2012]. Disponível: http://www.abcdt.org.br/ index.php?option $=$ com_content $\&$ view $=$ article\&id $=224:$ crianca-tambem-sofre-de-insuficiencia-renal\& catid=46:saude-em-destaque\&ltemid $=105$.

13. Hiep TTM, Ismaili K, Collart F, Damme-Lombaerts RV, Godefroid N, Ghuysen MS, et al. Clinical caracteristics and outcomes children with estage 3-5 chronic kidney disease. Pediatr. Nephrol. [Internet] 2010;25(5) [acesso em 15 out 2012]. Disponível: http://www.ncbi.nlm. nih.gov/pubmed/20148340. doi: 10.1007/s00467-0091424-2.

14. Peco-Antic A, Bogdanovic R, Paripovic D, Paripovic A, Kocev N, Golubovic E, et al. Epidemiology of chronic kidney disease in children in Serbia. Nephrol Dial Transplant. [Internet]. 2012;27(5):1978-84. [acesso em 16 out 2012]. Disponível: http://ndt.oxfordjournals.org/ content/27/5/1978.long. doi: 10.1093/ndt/gfr556.

15. Neu AM, Frankenfield DL. Clinical outcomes in pediatrics hemodialisys patients in the USA: lesson from CMS' ESRD CPM Project. Pediatr Nephrol. [Internet]. 2009;24(7) [acesso em 16 out 2012]. Disponível: http://www.ncbi.nlm.nih.gov/pmc/articles/ PMC2688608/pdf/467_2008_Article_831.pdf.
16. North American Pediatric Renal Trials and Collaborative Studies (NAPRTCS) [Internet]. 2011 Annual Dialysis Report. [acesso em 18 jul 2013]. Disponível: https:// web.emmes.com/study/ped/annlrept/annualrept2011. pdf.

17. Souza RA, Olivera EA, Silva JMP, Lima EM. Avaliação do acesso vascular para hemodiálise em crianças e adolescentes: um estudo de coorte retrospectivo de 10 anos. J. Bras. Nefrol. [Internet]. 2011;33(4) [acesso em 16 out 2012]. Disponível: http://dx.doi.org/10.1590/ S0101-28002011000400006

18. Santos AA, Siqueira CC, Sória DAC. Padronização dos cuidados com acessos vasculares para terapia hemodialítica: cuidado essencial de enfermagem. R. Pesq.: cuid. fundam. online. [Internet]. 2010;2(Suppl.) [acesso em 16 out 2012]. Disponível: http://www. seer.unirio.br/index.php/cuidadofundamental/article/ view/1061/pdf_226.

19. Aguiar NTR, Ferreira CD, Viviani J, André KM, Lopes $\mathrm{VM}$. The care of nurses in maintenance of arteriovenous fistula (fav). R. Pesq.: cuid. fundam. online. [Internet]. 2011;3(4) [acesso em 16 out 2012]. Disponível: http:// www.seer.unirio.br/index.php/cuidadofundamental/ article/view/1332/pdf_453. 